1

3

4

5

6

7

8

9

10

11

12

13

14

15

16

17

18

19

20

21

22

23

\title{
Clinical Features and Management of Eyelid Malignancies
}

Omotayo A. Arowojolu, M.D., Ph.D. ${ }^{1}$, Sanja G. Cypen, M.D. ${ }^{2}$, Jeremiah P. Tao, M.D., F.A.C.S. ${ }^{2}$

${ }^{1}$ Department of Plastic Surgery, University of California, Irvine, Orange, California, U.S.A.

${ }^{2}$ Gavin Herbert Eye Institute, University of California, Irvine, Irvine, California, U.S.A.

Corresponding author: Sanja G. Cypen, M.D.

Department of Ophthalmology, Gavin Herbert Eye Institute

University of California, Irvine

850 Health Sciences Road, Irvine, California, 92697, U.S.A.

Keywords: eyelid lesions, periocular, biopsy, eyelid malignancy

Financial support: None

scypen@hs.uci.edu

Conflicts of interest: The authors have no financial or conflicts of interest to disclose.

Received: June 162020

Accepted: December 15, 2020

Published online: December 23, 2020

This manuscript has been accepted for publication but not yet copyedited or typeset, and may be subject to minor changes during the production process. 


\section{Abstract}

Eyelid lesions are common. Pattern recognition and knowledge of common characteristics aid in

27 the assessment for malignancy. Ulceration, irregularity, telangiectasia, pearly appearance, and

28 loss of eyelid margin architecture suggest malignancy. The authors review these signs and

29 detail some common eyelid malignancies and treatment options for more advanced disease

30 including novel targeted therapies.

\section{Introduction}

The eyelids may harbor malignancies that can pose serious risks to the eye. Some carcinomas

34 can be life-threatening or associated with distant metastasis. More commonly, failure to

35 diagnose and treat in a timely manner can lead to local tumor growth with possible direct extension to the adjacent ocular surface, orbit, or skull base. Late treatment may require extensive resection and reconstruction.

Early detection of eyelid cancer is key. Distinguishing benign from malignant periocular lesions can be challenging. A patient's past medical history, as well as an assessment for characteristic signs, helps lead to a suspicion for malignancy, a decision for biopsy, and a definitive diagnosis.

42 In this review, the authors describe common clinical signs of eyelid malignancies and detail

43 features of some of the more common carcinomas found in the periocular region. We also describe treatments, including novel targeted therapies, for some aggressive periocular cancers. 


\section{Patient history}

The timeline of symptoms may aid in distinguishing a new, rapidly growing lesion from a longstanding lesion with recent transformation. Other symptoms, such as bleeding, crusting, non-healing, or recurrence, should prompt further work-up. Risk factors for cutaneous eyelid malignancies include advanced age, significant sun exposure with sunburns or prior skin cancer, prior radiation therapy (RT), or immunosuppression. A personal history of prior skin cancer or also a family history of skin cancer, particularly melanoma, may be important.

\section{Characteristics of eyelid malignancy}

Ulceration, irregular borders, telangiectasia, pearly borders, and loss of eyelid margin architecture (Table 1) are key features suggestive of eyelid malignancy. In particular, these findings are associated with periocular non-melanoma skin cancers, namely basal cell carcinoma and squamous cell carcinoma. ${ }^{1}$ Asymmetry, border, color, diameter, and evolution $(A B C D E)$, are the classic features to ascertain of pigmented skin lesions concerning for melanoma. $^{2}$

\section{Ulceration}

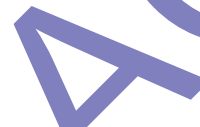

Ulceration develops as malignant cells grow haphazardly and are thought to outgrow their own blood supply (Figure 1A). With nodular basal cell carcinoma (BCC), tumors are perfused peripherally; however, central ischemia and ulceration develop. In squamous cell carcinoma (SCC), ulceration may be seen at the periphery of the lesion. In melanoma, ulceration suggests a more aggressive subtype or advanced disease. Ulceration, particularly chronic and recurrent, should prompt biopsy, as this feature is rarely seen in benign lesions. One exception is 
70 molluscum contagiosum, benign viral lesions that may present with chronic ulceration at the site

71 of central umbilication.

72

73 Irregularity

74 Irregularity in the contour or borders of an eyelid lesion occurs due to the presence of multiple

75 cellular populations growing at different rates and should raise suspicion for malignancy (Figure

76 1B). Malignant tumors often have scalloped margins with asymmetric shapes, whereas benign

77 lesions, such as syringomas, epidermal cysts, and intradermal nevi, typically have smooth,

78 discrete borders with a symmetric shape.

79

80

Telangiectasia

81 Telangiectasias are dilated, irregular small blood vessels of the superficial dermis (Figure 1C).

82 They are present in sun-exposed areas of the skin in older, fair-skinned individuals and are

83 associated with numerous cutaneous pathologies, including rosacea and scleroderma.

84 Dermatoscopic studies have shown that the pattern of arborizing telangiectasias is consistent

85 with nodular BCC on the face compared to the spoke-wheel, fine telangiectasias associated

86 with superficial $\mathrm{BCC} .{ }^{3}$ While isolated telangiectasias are commonly seen on older, sun-damaged

87 skin, telangiectasias overlying raised, scaly, or irregular skin lesions should raise suspicion for

88 an underlying skin cancer.

89

90

Pearly appearance 
91 A whitish and shiny appearance of a lesion may be suspicious. In particular, nodular BCC

92 presents with a scalloped pearly border and translucent appearance secondary to proliferating cells in the basal epidermis (Figure 1D). The differential diagnosis of pearly appearance may also include benign lesions, such as amelanotic nevi, papilloma, or molluscum contagiosum, among other less common entities.

Erosion of the mucocutaneous junction, a focal depression or notch, or other obliteration of eyelid margin landmarks, such as the meibomian gland orifices or lash line, are suspicious (Figure 1E). Madarosis, or loss of the eyelashes, is common with malignancy due to destruction of hair follicles, particularly with lesions that disrupt the normal eyelid margin architecture. As the tumor outgrows its blood supply, necrosis may ensue with cycles of granulation and healing. Eyelid margin destruction is particularly characteristic of morpheaform BCC or SCC and should encourage prompt biopsy.

\section{Basal cell carcinoma}

$\mathrm{BCC}$ is the most common cutaneous malignancy, comprising $90 \%$ of eyelid malignancies and approximately $20 \%$ of all eyelid tumors in general. ${ }^{4}$ Risk factors include ultraviolet (UV) exposure, specifically intermittent intense UVB, and fair skin. The association with sunburns suggests intensity of light, rather than prolonged sun exposure, predisposes individuals to BCC.

111 Nodular BCC may lead to what has been described as a "rodent ulcer," due to a destructive,

112 "eaten" appearance, which comprises half of all BCCs. The classic appearance, especially early 113 on, is a pink, pearly papule with a smooth translucent surface and telangiectasias. Additional 
114 features include margins that are difficult to discern, indurated texture, and central ulceration.

115 Common high-risk features include large lesion size and proximity to the medial canthus. ${ }^{5,6}$

116 Pigmented BCCs are a brown or black-blue hue and are distinguished from melanoma on

117 histopathology. Morpheaform BCC is more invasive with a worse prognosis. ${ }^{6}$ These lesions may

118 mimic a scar and have less discrete borders and skip lesions.

If clinical features are suspicious for malignancy, a biopsy should be performed. Incisional

121 biopsy is useful for large lesions that will require subsequent wide local resection with or without

122 Mohs micrographic surgery (MMS) followed by reconstructive surgery. Otherwise, smaller

123 lesions may be amenable to excisional biopsy with generous margins, permanent sections, and concurrent reconstruction to avoid multiple surgical sessions.

BCC most commonly involves the lower eyelid due to greatest direct sun exposure, followed by

127 the medial canthus, upper eyelid, and lateral canthus? If the lesion involves the medial canthus, 128 magnetic resonance imaging (MRI) of the orbits should be considered to evaluate for orbital extension. ${ }^{8}$

\section{Squamous cell carcinoma}

SCC is less common than BCC, comprising $5-10 \%$ of eyelid malignancies with a BCC to SCC

133 ratio of 4 to $1.6,9$ SCC occurs in sun-damaged skin and may arise de novo or from actinic 134 keratosis. The latter results from atypical keratinocyte proliferation and is classified as a premalignant lesion, thus should be monitored closely for characteristics suggestive of malignant transformation. Keratoacanthoma, also a precursor to SCC, is characterized by rapid onset (2-3 
137 weeks) of a crater-like lesion with central ulceration and raised erythematous borders. ${ }^{10,11}$

138 Seborrheic keratosis is a "greasy" "stuck-on" lesion on the differential for SCC, but is benign.

139 These lesions are typically seen in elderly patients and can be removed if causing irritation.

141 SCC may appear clinically as scaly skin, cutaneous horns, and rough patches. ${ }^{12,13}$ Additionally,

142 ulceration, telangiectasias, and erythema may be present along the margins and should prompt

143 biopsy. ${ }^{6}$ On histopathology, classic findings include hyperkeratosis and keratin deposits. SCC is

144 typically more aggressive than BCC and, when in advanced stages, may have perineural

145 invasion that is challenging to completely resect.

147 Sebaceous cell carcinoma

148 Sebaceous cell carcinomas (SBCC) are rare, comprising $<1$ to $5 \%$ of eyelid cancers. ${ }^{16}$ These

149 are aggressive tumors that arise within sebaceous glands of the eyelids and are potentially

150 lethal. They have no characteristic appearance, but may present as chronic

151 blepharoconjunctivitis or a recurrent chalazion. 17 Therefore, any chalazion recurring in the same

152 location should be investigated. A full-thickness biopsy is usually required to make the

153 diagnosis. The sample is usually sent as a fresh specimen; permanent fixation with formalin

154 may wash the lipoid component and tarnish the diagnostic yield. SBCC can have skip lesions;

155 hence, a wide resection should be performed. Some experts recommend conjunctival map

156 biopsies to assess for skip lesions, particularly in cases with multifocal masses on gross

157 examination. ${ }^{18}$ Sentinel lymph node biopsy (SLNB) can be considered, although the effect on

158 survival outcomes is unclear. ${ }^{19}$ Orbital exenteration is necessary in some aggressive cases with

159 orbital extension to prevent the risk of intracranial extension and metastasis (Figure 2). 
162 Eyelid melanoma is rare, making up 1\% of all malignant neoplasms of periocular skin. ${ }^{20}$ It 163 occurs in one of three forms: superficial spreading malignant melanoma (MM), lentigo maligna 164 melanoma (LMM), and nodular melanoma (NM). LMM is typically non-palpable with tan to 165 brown pigmentation and irregular margins; however, darker and elevated lesions occur with 166 dermal invasion. NM is a slightly raised blue-black lesion but can also be amelanotic. MM has

167 potential for distant metastasis to areas including the central nervous system, bone, and 168 gastrointestinal tract and is often lethal. ${ }^{21,22}$ The risk and prognosis correlate directly with 169 Breslow depth, which can be obtained through early incisional biopsy of pigmented lesions demonstrating changes in size, color, or shape. Metastasis occurs through lymphatic spread;

171 therefore, regional nodal status is the most important prognostic factor. ${ }^{23,24}$ Consideration 172 should be given to performing SLNB concurrently with surgical excision, particularly in lesions 173 with Breslow thickness greater than $0.8 \mathrm{~mm}$ or with ulceration. ${ }^{25,26}$ For intermediate thickness 174 melanoma, SLNB has been associated with improved survival in controlled studies. The 175 differential for pigmented lesions includes benign lesions, such as nevi, verruca, papillomas, 176 and solar lentigos. These lesions can be irritating or pruritic in contrast to the indolent 177 presentation of melanoma.

\section{Merkel cell carcinoma}

Merkel cell carcinoma (MCC) is rare but increasing in frequency due to improved diagnosis and detection. MCC is a highly malignant neuroendocrine tumor with worse prognosis in the head and neck compared to other sites. ${ }^{27} \mathrm{MCC}$ is associated with age greater than 50 years and

183 immunosuppression, including human immunodeficiency virus and organ transplantation. The 
184 tumor presents as a painless, rapidly growing, non-tender, red or violaceous nodule, with

185 difficult to discern borders. It is aggressive with high incidence of regional metastasis. Therefore,

186 wide tumor resection with 1-2 centimeter margins is recommended, and SLNB may be

187 considered to detect regional metastasis. ${ }^{27}$

\section{Biopsy techniques}

190

191

192

193

194

Incisional biopsy

An incisional biopsy involves removal of part of the lesion through a shave or punch biopsy technique. If the lesion is confirmed to be benign, an incisional biopsy is advantageous as it produces an aesthetic result with minimal surgical intervention and minimal distortion of the adjacent tissue. Further, it is advantageous for larger, high risk lesions, as it allows quick and convenient tissue sampling to aid further surgical planning and coordination among specialists (e.g., Mohs surgeon or surgical oncologist). Conversely, the disadvantage of incisional biopsy is that it requires an additional surgical procedure if the final histopathology reveals malignancy. ${ }^{28}$ Nevertheless, incisional biopsy allows sampling of the lesion and can usually be performed in a timely manner as an in-office procedure under local anesthesia.

\section{Excisional biopsy}


206 Excisional biopsy involves entire removal of all grossly visible tumor. ${ }^{29}$ For eyelid lesions, full

207 thickness excisional biopsies may completely treat lesions close to the eyelid margin. Medial

208

209

210

211

212

213

214

215

216

217

219

220

221

222

223

224

225

226

227

228

229 eyelid tumors should be resected with caution due to their proximity to the canaliculi. Concurrent nasolacrimal stenting and repair of the canaliculi may be warranted. For excisional biopsy of a non-superficial eyelid margin lesion, a full thickness pentagonal wedge excision with appropriate margins may achieve excellent aesthetic and functional results. The apex should extend through the entirety of the tarsus to avoid eyelid contour abnormalities including notching and kinking. ${ }^{30}$ Despite careful approximation of the eyelid edges, the vertical wound results in a conspicuous scar and the triangular apex can have skin redundancy or form a "dog-ear". A curvilinear or "lazy" pentagonal wedge excision design may achieve tarsal alignment while reducing cutaneous redundancy and keep the incision aligned with the relaxed facial skin tension lines. ${ }^{31}$

\section{Diagnostic Studies}

Adjunctive diagnostic studies may aid in diagnosis and staging of advanced cutaneous malignancies and should be obtained in specific circumstances. Pre-operative neuroimaging of the orbits with MRI or computed tomography (CT) should be obtained if the affected eye has abnormal conjunctival fornix architecture or if orbital signs are present, including vision changes, restriction of extraocular movements, proptosis, or pupil abnormality.

MRI is more sensitive for evaluating the orbital soft tissues - the primary pattern of invasion for most eyelid malignancies. However, CT is useful if bony erosion is suspected. CT is also indicated if there is a contraindication to MRI, such as a metallic implant, or if MRI is not readily available. For highly aggressive tumors with potential for regional metastasis, such as SBCC or 
$230 \mathrm{MCC}$, positron emission tomography (PET) may be indicated to highlight areas of high

231 metabolic activity throughout the body suggestive of metastases.

233 Treatment Options

234 Treatment options for biopsy-proven eyelid and periocular malignancies vary based upon the 235 malignancy and depth of invasion. As aforementioned, excisional biopsy itself may be curative.

236 For biopsies with positive tumor margins, wide local excision with confirmation of negative

237 surgical resection margins is the gold standard. Cryo-, thermo-, or local chemo-therapy are 238 options, especially if a patient cannot tolerate surgery. However, these options are less 239 desirable owing to proximity to the eye. Importantly, tumor eradication is not histologically 240 validated with these tissue destruction options. Hence, surgical resection with intraoperative 241 frozen sections or MMS to evaluate margins prior to reconstruction in the same or separate 242 surgical setting is preferable. Incomplete tumor excision remains a small risk even with MMS or

245 For aggressive tumors, especially if the margins remain positive, globe-sparing medical oncology treatment options should be considered when appropriate. Neoadjuvant, adjuvant, and

247 radiation therapies may not eradicate disease but may reduce tumor size burden to improve the opportunity for surgical resection.

250 When globe-sparing options are futile or if the globe and vision are already compromised, 251 locally advanced tumors confined to the orbit can be treated with orbital exenteration. In these 252 procedures periocular and orbital contents, including the eyeball, orbital soft tissues, lacrimal 
253 gland, lacrimal drainage apparatus, periosteum, and eyelids are removed en bloc. This

254 procedure is potentially lifesaving but notwithstanding disfiguring side effects.

255

256 Surgical oncology specialty involvement may be useful for local tumor resection or concomitant

257 SLNB or both, particularly in cases of SBCC, melanoma, Merkel cell carcinoma, or invasive

258 SCC with high-risk features. However, the role of SNLB in malignancies besides intermediate-

259 thickness melanoma is yet to be defined. ${ }^{6,19}$

260

261 Targeting cell signaling pathways involved in eyelid tumor pathogenesis is a new paradigm for 262 certain invasive cancers, particularly those that are not amenable to surgical resection or to 263 avoid orbital exenteration. ${ }^{33}$

265 Targeting the aberrantly activated sonic hedgehog $(\mathrm{SHH})$ signaling pathway has been

266 associated with improved clinical response in patients with advanced BCC. Vismodegib

267 deactivates the SHH signaling pathway through inhibition of the Smoothened (SMO) receptor. ${ }^{33}$

268 It is currently indicated for BCC that is locally advanced, metastatic, recurrent, or not amenable

269 to surgery or RT. ${ }^{34}$ Moreover, when used in combination with mammalian target of rapamycin

270 (mTOR) inhibitors and RT, surgical resection has been found to be less extensive. ${ }^{35}$ Its use as

271 neoadjuvant therapy with the goal of globe-sparing surgery is under investigation.

273 Overexpression of epidermal growth factor receptor (EGFR) has been shown in SCC

274 pathogenesis. Thus, targeted therapies for non-resectable, metastatic or recurrent SCC of the

275 head and neck include EGFR inhibitors and immune checkpoint inhibitors. Erlotinib, FDA- 
276 approved for use in advanced non-small cell lung and pancreatic cancers, is a tyrosine kinase

277 EGFR antagonist used off-label for advanced periorbital SCC. ${ }^{33}$ Cetuximab is a monoclonal

278 antibody EGFR antagonist used in combination with RT for locoregionally advanced tumors or

279 with platinum-based chemotherapy for recurrent/metastatic cutaneous SCC. It has also been

280

281

282

283

284

285

286

287

288

289

290

291

292

293

294

295

296

297

298

299 used as neoadjuvant therapy to chemo-reduce tumor burden and improve surgical outcomes. ${ }^{36}$ Immune checkpoint inhibitors, include pembrolizumab and cemiplimab. Pembrolizumab, a programmed cell death protein 1 (PD-1) receptor inhibitor, has been associated with regression of a massive, non-resectable SCC. ${ }^{37}$ Migden and colleagues report that in patients with advanced cutaneous SCC, PD-1 blockade with cemiplimab showed a response rate of $47 \%$ in patients with metastatic disease. ${ }^{38}$ The role of these treatments may be incompletely understood presently, but these therapies demonstrate a potential to augment or possibly replace aggressive surgical resection.

Cutaneous melanomas have demonstrated clinical response with targeted molecular therapy. Fifty percent of melanomas have BRAF gene mutations with $90 \%$ of these cases being the V600E gene mutation, which leads to persistent activation of the MAPK signaling pathway. Vemurafenib is a BRAF inhibitor used for metastatic or non-resectable melanoma with the V600E mutation. Dabrafenib, a BRAF inhibitor, is used with trametinib, a MEK inhibitor, for V600E and V600K mutations, as resistance develops through activation of the MEK signaling pathway. ${ }^{39}$ Currently, there are no reports of BRAF inhibitors for treatment of eyelid melanomas. However, conjunctival melanoma has demonstrated improved clinical outcomes with promising long-term results. ${ }^{40}$ Immune checkpoint inhibitors for cutaneous melanoma include nivolumab (PD-1 inhibitor) for metastatic or non-resectable melanomas, pembrolizumab for metastatic melanoma, and ipilimumab (CTLA-4 inhibitor) for metastatic and non-resectable melanoma. 
301 The primary treatment for SBCC is wide surgical excision, although pembrolizumab has been

302 used off-label for advanced stage or recurrent sebaceous carcinoma. ${ }^{41}$ For advanced and

303 metastatic MCC, avelumab, an anti-PD ligand-1, has demonstrated clinical response. ${ }^{42}$

304 Pembrolizumab and nivolumab have also been shown to improve survival in advanced and

305 metastatic MCC. ${ }^{42}$ However, no specific studies have investigated their efficacy in eyelid or

306 periocular MCC.

307

308

Conclusion

309

Eyelid cancer is common, with BCC being the most prevalent. ${ }^{4,6}$ Ulceration, irregularity,

310 telangiectasia, pearly appearance, and loss of eyelid margin architecture are key features

311 suspicious for malignancy. The final diagnosis rests with tissue biopsy. Surgical excision with

312 negative margins remains the gold standard therapy, although newer therapies are available for

313 advanced cases or when surgical resection is not possible. Targeted therapies are promising for

314 aggressive or advanced periocular cancers. 


\section{Table and Figure Legends}

317 Table 1. Characteristic features of malignant periocular cutaneous lesions

318 Figure 1. Characteristics of periocular skin cancers include: ulceration (A), irregularity (B),

319 telangiectasia (C), pearly appearance (D), and loss of eyelid margin architecture (E).

320 Figure 2. Magnetic resonance imaging (MRI) scan of the orbits ( $T 1$, post-contrast with fat

321 suppression) demonstrating orbital invasion of a biopsy-proven sebaceous cell carcinoma. A.

322 Axial scan demonstrating an enhancing mass (arrow) of the lateral orbit abutting the globe and

323 resulting in medial displacement of the globe. B. Coronal scan demonstrating an enhancing

324 mass circumferentially surrounding the globe (arrow) with possible extension into the lacrimal

325 sac (asterisk). 


\section{References}

328

329

330

331

332

333

334

335

336

337

338

339

340

341

342

343

344

345

346

347

348

349

350

351

352

353

354

355

356

357

358

359

1. Loeffler M, Hornblass A. Characteristics and behavior of eyelid carcinoma (basal cell, squamous cell sebaceous gland, and malignant melanoma). Ophthalmic Surg. 1990;21(7):513-518.

2. Tsao H, Olazagasti JM, Cordoro KM, et al. Early detection of melanoma: reviewing the ABCDEs. J Am Acad Dermatol. 2015;72(4):717-723.

3. Suppa M, Micantonio T, Di Stefani A, et al. Dermoscopic variability of basal cell carcinoma according to clinical type and anatomic location. J Eur Acad Dermatol Venereol. 2015;29(9):1732-1741.

4. Tao J, Wachter B. Basal Cell Carcinoma of Eyelid. In: Schmidt-Erfurth U, Kohnen T, eds. Encyclopedia of Ophthalmology. Berlin, Heidelberg: Springer Berlin Heidelberg; 2018:225-227.

5. Miller SJ, Alam M, Andersen J, et al. Basal cell and squamous cell skin cancers J Nat/ Compr Canc Netw. 2010;8(8):836-864.

6. Gandhi SA, Kampp J. Skin Cancer Epidemiology, Detection, and Management. Med Clin North Am. 2015;99(6):1323-1335.

7. Cook BE, Jr., Bartley GB. Epidemiologic characteristics and clinical course of patients with malignant eyelid tumors in an incidence cohort in Olmsted County, Minnesota. Ophthalmology. 1999;106(4):746-750.

8. Leibovitch I, McNab A, Sullivan T, Davis G, Selva D. Orbital invasion by periocular basal cell carcinoma. Ophthalmology. 2005;112(4):717-723.

9. Tao J, Wachter B. Squamous Cell Carcinoma of Eyelid. In: Schmidt-Erfurth U, Kohnen T, eds. Encyclopedia of Ophthalmology. Berlin, Heidelberg: Springer Berlin Heidelberg; 2018:1664-1666.

10. Boniuk M, Zimmerman LE. Eyelid tumors with reference to lesions confused with squamous cell carcinoma. 3. Keratoacanthoma. Arch Ophthalmol. 1967;77(1):29-40.

11. Grossniklaus HE, Wojno TH, Yanoff M, Font RL. Invasive keratoacanthoma of the eyelid and ocular adnexa. Ophthalmology. 1996;103(6):937-941.

12. Pyne J, Sapkota D, Wong JC. Cutaneous horns: clues to invasive squamous cell carcinoma being present in the horn base. Dermatol Pract Concept. 2013;3(2):3-7.

13. Mencia-Gutierrez E, Gutierrez-Diaz E, Redondo-Marcos I, Ricoy JR, Garcia-Torre JP. Cutaneous horns of the eyelid: a clinicopathological study of 48 cases. J Cutan Pathol. 2004;31(8):539-543.

14. McNab AA, Francis IC, Benger R, Crompton JL. Perineural spread of cutaneous squamous cell carcinoma via the orbit. Clinical features and outcome in 21 cases. Ophthalmology.

1997;104(9):1457-1462. 
360

361

362

363

364

365

366

367

368

369

370

371

372

373

374

375

376

377

378

379

380

381

382

383

384

385

386

387

388

389

390

391

392

393 394

15. Limawararut V, Leibovitch I, Sullivan T, Selva D. Periocular squamous cell carcinoma. Clin Exp Ophthalmol. 2007;35(2):174-185.

16. Tao J, Wachter B. Sebaceous Carcinoma/Adenocarcinoma. In: Schmidt-Erfurth U, Kohnen T, eds. Encyclopedia of Ophthalmology. Berlin, Heidelberg: Springer Berlin Heidelberg; 2018:1603-1604.

17. Shields JA, Demirci H, Marr BP, Eagle RC, Jr., Shields CL. Sebaceous carcinoma of the eyelids: personal experience with 60 cases. Ophthalmology. 2004;111(12):2151-2157.

18. Buitrago W, Joseph AK. Sebaceous carcinoma: the great masquerader: emgerging concepts in diagnosis and treatment. Dermatol Ther. 2008;21(6):459-466.

19. Freitag SK, Aakalu VK, Tao JP, et al. Sentinel Lymph Node Biopsy for Eyelid and Conjunctival Malignancy: A Report by the American Academy of Ophthalmology. Ophthalmology 2020;127(12):1757-1765.

20. Tao J, Wachter B. Melanoma of the Eyelid. In: Schmidt-Erfurth U, Kohnen T, eds. Encyclopedia of Ophthalmology. Berlin, Heidelberg: Springer Berlin Heidelberg; 2018:1123-1125.

21. Cook BE, Jr., Bartley GB. Treatment options and future prospects for the management of eyelid malignancies: an evidence-based update. Ophthalmology. 2001,108(11):2088-2098; quiz 20992100, 2121.

22. Boulos PR, Rubin PA. Cutaneous melanomas of the eyelid. Semin Ophthalmol. 2006;21(3):195206.

23. Balch CM, Soong SJ, Gershenwald JE, et al. Prognostíc factors analysis of 17,600 melanoma patients: validation of the American Joint Committee on Cancer melanoma staging system. J Clin Oncol. 2001;19(16):3622-3634.

24. Mendoza PR, Grossniklaus HE. Sentinel lymph node biopsy for eyelid and conjunctival tumors: what is the evidence? Int Ophthalmol Clin. 2015;55(1):123-136.

25. Savar A, Ross MI, Prieto VG, Ivan D, Kim S, Esmaeli B. Sentinel lymph node biopsy for ocular adnexal melanoma: experience in 30 patients. Ophthalmology. 2009;116(11):2217-2223.

26. National Comprehensive Cancer $\mathbf{N}$. Cutaneous Melanoma (Version 2.2020). https://www.nccn.org/professionals/physician gls/pdf/cutaneous melanoma.pdf. Published 2020. Accessed April 25, 2020.

27. Muller-Richter UDA, Gesierich A, Kubler AC, Hartmann S, Brands RC. Merkel Cell Carcinoma of the Head and Neck: Recommendations for Diagnostics and Treatment. Ann Surg Oncol. 2017;24(11):3430-3437.

28. Tao J, Wachter B, Echegoyen J. Incisional Biopsy. In: Schmidt-Erfurth U, Kohnen T, eds. Encyclopedia of Ophthalmology. Berlin, Heidelberg: Springer Berlin Heidelberg; 2018:923-924.

29. Tao J, Wachter B, Echegoyen J. Excisional Biopsy. In: Schmidt-Erfurth U, Kohnen T, eds. Encyclopedia of Ophthalmology. Berlin, Heidelberg: Springer Berlin Heidelberg; 2018:747-748. 
395

396

397

398

399

400

401

402

403

404

405

406

407

408

409

410

411

412

413

414

415

416

417

418

419

420

421

422

423

30. Tao J, Wachter B, Echegoyen J. Full-Thickness Eyelid Biopsy. In: Schmidt-Erfurth U, Kohnen T, eds. Encyclopedia of Ophthalmology. Berlin, Heidelberg: Springer Berlin Heidelberg; 2018:790791.

31. Garcia GA, Nguyen CV, Vo TA, Yonkers MA, Minckler DS, Tao JP. Lazy Pentagonal Wedge Resection of Eyelid Margin Lesions. JAMA Facial Plast Surg. 2018;20(3):251-252.

32. Lo JS, Snow SN, Reizner GT, Mohs FE, Larson PO, Hruza GJ. Metastatic basal cell carcinoma: report of twelve cases with a review of the literature. J Am Acad Dermatol. 1991;24(5 Pt 1):715719.

33. Mehta VJ, Ling J, Sobel RK. Review of Targeted Therapies for Periocular Tumors. Int Ophthalmol Clin. 2017;57(1):153-168.

34. FDA labeling information - ERIVEDGE [press release]. FDA website, 20192012.

35. Sagiv O, Sa H-S, Esmaeli B. Basal cell carcinoma : advances in treatment and research. In: Migden MR, Chen L, Silapunt S, eds. Cham: Springer; 2020:161-176.

36. Sagiv O, Jaber B, Esmaeli B. Targeted and Immune Therapy for Periocular and Orbital Malignancies. In: Gupta A, Tahiliani P, eds. Orbit and Oculoplastics : Newer Trends. Singapore: Springer Singapore; 2019:83-97.

37. Conger JR, Grob SR, Tao J. Massive Periocular Squamous Cell Carcinoma With Response to Pembrolizumab (Keytruda). Ophthalmic Plast Reconstr Surg. 2019;35(5):e127.

38. Migden MR, Rischin D, Schmults CD, et al. PD-1 Blockade with Cemiplimab in Advanced Cutaneous Squamous-Cell Carcinoma. N Engl J Med. 2018;379(4):341-351.

39. Johannessen CM, Boehm JS, Kim SY, et al. COT drives resistance to RAF inhibition through MAP kinase pathway reactivation. Nature. 2010;468(7326):968-972.

40. Lu JE, Chang JR, Berry JL, In GK, Zhang-Nunes S. Clinical Update on Checkpoint Inhibitor Therapy for Conjunctival and Eyelid Melanoma. Int Ophthalmol Clin. 2020;60(2):77-89.

41. Lin AC, Shriver EM. The Role of Pembrolizumab in the Treatment of Sebaceous Carcinoma. Int Ophthalmol Clin. 2020;60(2):39-46.

42. Garcia GA, Kossler AL. Avelumab as an Emerging Therapy for Eyelid and Periocular Merkel Cell Carcinoma. Int Ophthalmol Clin. 2020;60(2):91-102. 
425 Figure 1:
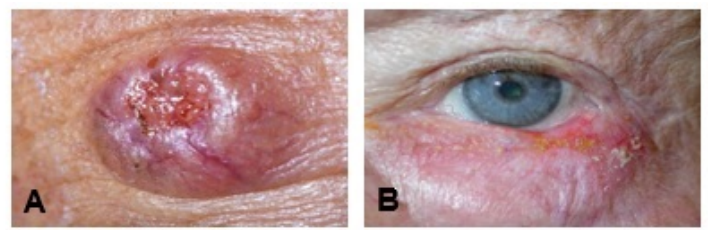

426
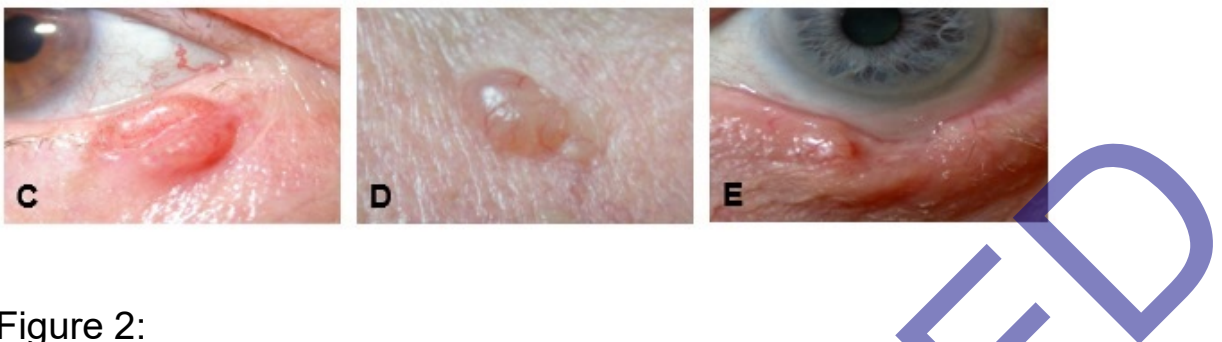

$427 \quad$ Figure 2:

428
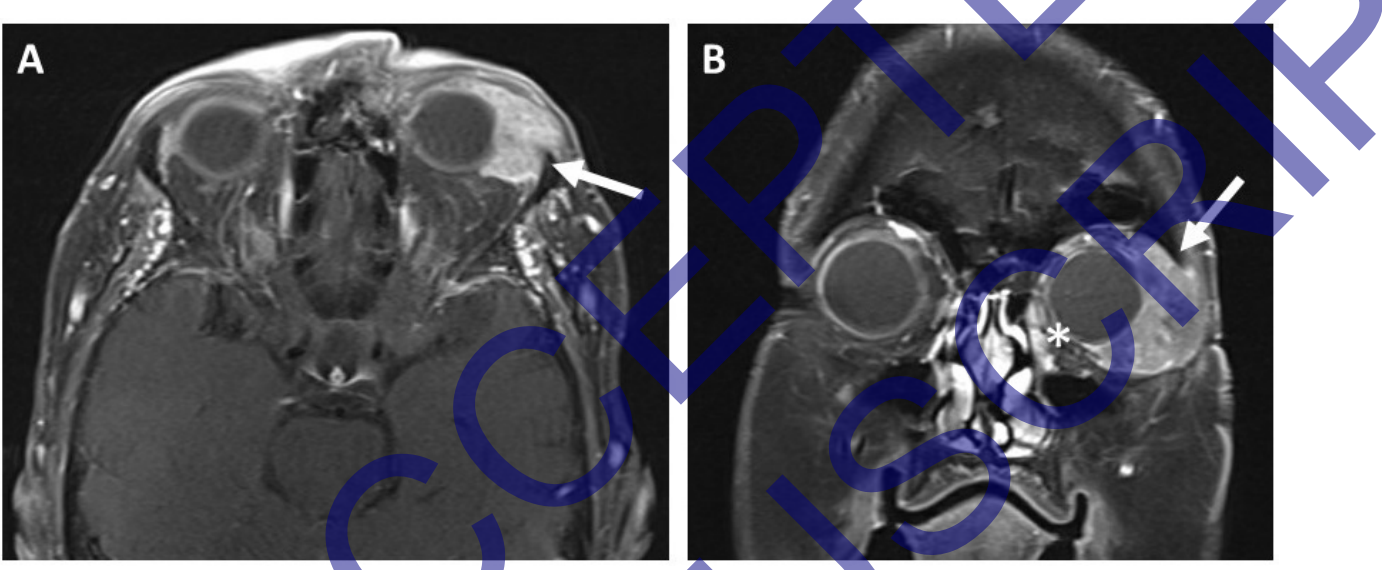

429

Table 1:

\begin{tabular}{l|l}
\multicolumn{1}{c}{ Feature } & \multicolumn{1}{c}{ Description } \\
\hline Ulceration & $\begin{array}{l}\text { Malignant cells outgrow their blood supply } \\
\text { resulting in central ischemia and ulceration }\end{array}$ \\
\hline Irregular borders & $\begin{array}{l}\text { Multiple cellular populations grow at different } \\
\text { rates resulting in asymmetric shapes along the } \\
\text { border }\end{array}$ \\
\hline Telangiectasias & $\begin{array}{l}\text { Arborizing, irregular blood vessels in the } \\
\text { superficial dermis }\end{array}$ \\
\hline Pearly appearance & $\begin{array}{l}\text { Proliferating cells in the basal epidermis result } \\
\text { in ivory, translucent appearance of cutaneous } \\
\text { lesions }\end{array}$ \\
\hline $\begin{array}{l}\text { Loss of eyelid margin } \\
\text { architecture }\end{array}$ & $\begin{array}{l}\text { Erosion of mucocutaneous junction, obliteration } \\
\text { of eyelid margin landmarks, or madarosis }\end{array}$
\end{tabular}

